\title{
Thermal expansion and phase transitions of $\alpha-\mathrm{AlF}_{3}$
}

\author{
Cody R. Morelock ${ }^{\mathrm{a}}$, Justin C. Hancock ${ }^{\mathrm{a}}$, and Angus P. Wilkinson ${ }^{\mathrm{a}, \mathrm{b}, *}$ \\ ${ }^{a}$ School of Chemistry and Biochemistry, Georgia Institute of Technology, Atlanta, GA 30332-0400, USA \\ ${ }^{b}$ School of Materials Science and Engineering, Georgia Institute of Technology, Atlanta, GA 30332-0245, USA \\ *Corresponding author: (404) 894 4036, angus.wilkinson@chemistry.gatech.edu
}

Keywords: Thermal expansion, rhenium trioxide, $\mathrm{ReO}_{3}$, aluminum fluoride, $\alpha-\mathrm{AlF}_{3}$, high temperature diffraction

\section{Graphical abstract:}

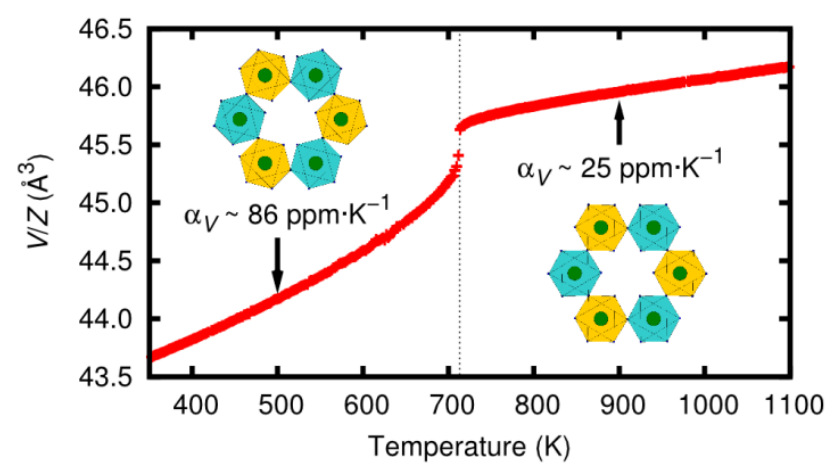

$\alpha-\mathrm{AlF}_{3}$ has a rhombohedrally distorted $\mathrm{ReO}_{3}$-type structure at ambient conditions and displays strongly positive volume thermal expansion that is highly anisotropic; the material becomes cubic on heating above $\sim 713 \mathrm{~K}$ and continues to show positive thermal expansion. 


\begin{abstract}
$\mathrm{ReO}_{3}$-type materials are of interest for their potential low or negative thermal expansion. Many metal trifluorides $M \mathrm{~F}_{3}$ adopt the cubic form of this structure at elevated temperatures, which rhombohedrally distorts upon cooling. The rhombohedral form displays strong positive volume thermal expansion, but cubic $M \mathrm{~F}_{3}$ display much lower and sometimes negative thermal expansion. The expansion behavior of $\alpha-\mathrm{AlF}_{3}$ was characterized via synchrotron powder diffraction between 323 and $1177 \mathrm{~K} . \alpha-\mathrm{AlF}_{3}$ is rhombohedral at ambient conditions and displays strongly anisotropic thermal expansion. The volume coefficient of thermal expansion (CTE), $\alpha_{V}$, at $500 \mathrm{~K}$ is $\sim 86 \mathrm{ppm} \cdot \mathrm{K}^{-1}$, but the linear $\mathrm{CTE}$ along the $c$-axis, $\alpha_{c}$, is close to zero. $\alpha-\mathrm{AlF}_{3}$ becomes cubic on heating to $\sim 713 \mathrm{~K}$ and continues to show positive thermal expansion above the phase transition $\left(\alpha_{V}(900 \mathrm{~K}) \sim 25 \mathrm{ppm} \cdot \mathrm{K}^{-1}\right)$.
\end{abstract}

\title{
1. INTRODUCTION
}

The simple cubic $\mathrm{ReO}_{3}$ structure (Figure 1), consisting of metal-centered octahedra that share anions at their corners, is of interest because of its potential for low or negative thermal expansion (NTE). This structure is related to that of perovskites, $A B X_{3}$, with a vacant $A$ site and hence significant empty volume, and has been used to illustrate how NTE can arise from the transverse thermal motion of bridging atoms and the resulting rocking of relatively rigid units. ${ }^{1,2}$ Rhenium trioxide itself displays low thermal expansion at ambient conditions, ${ }^{3-6}$ but members of this structural family, including fluorides and oxyfluorides, have coefficients of thermal expansion (CTE) ranging from strongly positive to strongly negative..$^{7-12}$ 


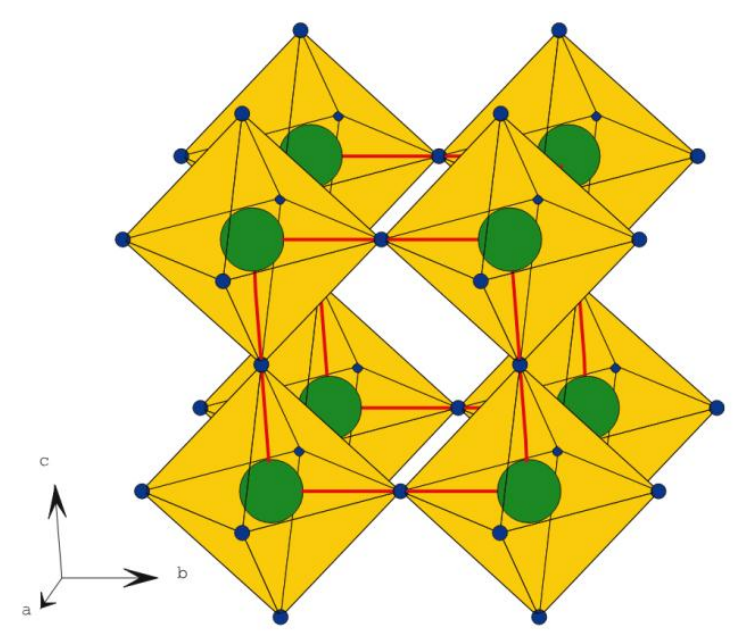

Figure 1. The cubic $\mathrm{ReO}_{3}$ structure, with the unit cell outlined in red. Green spheres represent metal ions, while blue spheres represent anions.

Many metal trifluorides $\left(M \mathrm{~F}_{3} ; M=\mathrm{Al}, \mathrm{Cr}, \mathrm{Fe}, \mathrm{Ga}, \mathrm{In}, \mathrm{Ti}, \mathrm{V}\right)$ are only cubic at elevated temperatures; upon cooling, they adopt a rhombohedrally distorted form of the $\mathrm{ReO}_{3}$ structure (VF-type). ${ }^{13-15}$ This phase transformation involves the coupled rotation of the constituent $M \mathrm{~F}_{6}$ octahedra. The thermal expansion of some $\mathrm{ReO}_{3}$-type $M \mathrm{~F}_{3}$ has been studied. The unfolding of the rhombohedral phase on heating is accompanied by strongly positive thermal expansion (PTE), while the cubic phase shows much lower or even negative thermal expansion. Notably, cubic $\mathrm{ScF}_{3}$ displays pronounced NTE over a wide temperature range (at least 10 to $1100 \mathrm{~K}$ ). ${ }^{10}$ $\mathrm{TiF}_{3}$ is cubic above $\sim 340 \mathrm{~K},{ }^{12,14,15}$ and its CTE is slightly positive just above the phase transition but then becomes slightly negative above $\sim 400 \mathrm{~K} .{ }^{12}$ We have recently explored the control of thermal expansion in $\mathrm{ScF}_{3}$-based materials by forming $\mathrm{Sc}_{1-x} M_{x} \mathrm{~F}_{3}(M=\mathrm{Ti}, \mathrm{Y})$ solid solutions. Solid solutions of $\mathrm{ScF}_{3}$ and $\mathrm{TiF}_{3}$ display CTEs ranging between those of the end members; ${ }^{12}$ however, in cubic $\mathrm{Sc}_{1-x} \mathrm{Y}_{x} \mathrm{~F}_{3}$, where the size mismatch between the two cations is quite large, the CTE is largely independent of composition. ${ }^{11}$ 
$\alpha-\mathrm{AlF}_{3}$ also has $\mathrm{ReO}_{3}$-type connectivity. At room temperature, the material is rhombohedral, becoming cubic at $\sim 730 \mathrm{~K} .{ }^{16}$ Although the rhombohedral-to-cubic phase transition in $\alpha-\mathrm{AlF}_{3}$ has been studied extensively, ${ }^{13,16-20}$ the thermal expansion of $\alpha-\mathrm{AlF}_{3}$ has not been reported in detail. In 1984, Ravez et al. provided some information on the temperature dependence of unit cell volume but did not report expansion coefficients; ${ }^{21}$ they reported that both cubic and rhombohedral phases display positive thermal expansion. In 2001, Chupas et al. studied $\alpha-\mathrm{AlF}_{3}$ in the context of $\gamma-\mathrm{Al}_{2} \mathrm{O}_{3}$ fluorination and catalysis. They reported that some weak reflections, potentially indicative of a residual rhombohedral distortion, persisted above the phase transition at $\sim 739 \mathrm{~K}$, and their Rietveld analyses suggested that bent $\mathrm{Al}-\mathrm{F}-\mathrm{Al}$ links persisted above the phase transition temperature. ${ }^{16} \mathrm{~A}$ later total scattering study of $\alpha-\mathrm{AlF}_{3}$ revealed that the cubic phase contains local octahedral tilts. ${ }^{20}$ The mechanism of the cubic-to-rhombohedral phase transition was studied using molecular dynamics simulations, and the authors suggested local distortions and NTE above the phase transition temperature. ${ }^{19}$ In the present paper, as part of our broader exploration of thermal expansion in $\mathrm{ReO}_{3}$-type fluorides, we reexamine in detail the anisotropic thermal expansion of $\alpha-\mathrm{AlF}_{3}$ between 323 and $1177 \mathrm{~K}$.

\section{MATERIALS AND METHODS}

\subsection{Sample preparation}

Anhydrous $\mathrm{AlF}_{3}$ (American Elements, 99.99\%) was ground thoroughly in an inert atmosphere and sealed in a nickel tube by welding. The Ni tube was then sealed in an evacuated fused quartz ampoule and heated at $1338 \mathrm{~K}$ for $72 \mathrm{~h}$, after which it was quenched by removing from the furnace. The heat-treated sample was maintained in an inert atmosphere to avoid moisture exposure. 


\subsection{Variable temperature synchrotron powder diffraction}

Variable temperature powder X-ray diffraction (PXRD) data $(\lambda \approx 0.7291 \AA)$ were collected at beamline 17-BM-B of the Advanced Photon Source (APS), Argonne National Laboratory, Illinois, USA. A PerkinElmer amorphous Si-based area detector $(2048 \times 2048,200 \mu \mathrm{m}$ pixels $)$ was used to record diffraction images, and $\mathrm{LaB}_{6}$ (NIST SRM 660a) was used to calibrate beam center, detector tilt angle and tilt plane rotation angle, as well as its distance to the sample. The $\alpha-\mathrm{AlF}_{3}$ powder sample was packed in a fused quartz capillary under argon, and UHP nitrogen was very slowly flowed through the capillary during data collection to avoid the ingress of any moisture. Temperature was controlled with a wire-wound furnace, ${ }^{22}$ heating at a rate of $180 \mathrm{~K} \cdot \mathrm{h}^{-1}$ from 323 to $1177 \mathrm{~K}$. No data were collected on cooling, as the sample capillary failed upon reaching the maximum temperature. Exposure times of $\sim 30 \mathrm{~s}$ were used, with a $\sim 30 \mathrm{~s}$ wait between exposures.

\subsection{Data analysis}

Two-dimensional area detector images were reduced to one-dimensional patterns with FIT2D. ${ }^{23}$ A calibration curve was created from the measured and expected peak positions for a standard $\mathrm{LaB}_{6}$ sample to correct for angular distortions in the 1D patterns that are believed to be associated with the oblique incidence of the X-ray beam on the relatively thick active layer of the

detector. Rietveld analyses were accomplished in the sequential refinement mode of $G S A S .{ }^{24,25} \mathrm{~A}$ fit to data at $323 \mathrm{~K}$ (Supporting Information, Figure S1) with a primitive rhombohedral model (space group $R 3 c$ ) served as the starting point for sequential refinement. An isotropic atomic displacement parameter (ADP) was refined for the $\mathrm{Al}$ atom, while anisotropic ADPs were used for the F atoms. Unit cell volumes per formula unit were extracted during Rietveld analyses and plotted with respect to temperature; the temperature variation of the volume CTE $\left(\alpha_{V}\right)$ was 
estimated by differentiation of sixth-order polynomial fits to these data. For some of the analyses, the primitive rhombohedral lattice constant $a_{R}$ and unit cell angle $\alpha_{R}$ extracted during Rietveld analyses were converted to hexagonal lattice constants $a_{H}$ and $c_{H}$ by the following relationships: $a_{H}=2 a_{R} \sin \frac{\alpha_{R}}{2}$ and $c_{H}=a_{R} \sqrt{3+6 \cos \alpha_{R}}$.

\section{RESULTS AND DISCUSSION}

The appearance of superlattice peaks and the splitting of other peaks at low temperatures in the variable temperature PXRD patterns (Figure 2) are evidence for the rhombohedral-to-cubic phase transition previously observed in $\alpha-\mathrm{AlF}_{3}{ }^{21}$ and other $\mathrm{ReO}_{3}$-type metal trifluorides. ${ }^{11,12,14,15}$ The phase transition appears quite clearly just above $700 \mathrm{~K}$ (Figure 2). We also observe an $\alpha$ $\mathrm{Al}_{2} \mathrm{O}_{3}$ phase, an impurity likely incorporated into the sample during the annealing process; the $\alpha$ $\mathrm{Al}_{2} \mathrm{O}_{3}$ peaks exist over the entire temperature range but are quite small $(<1 \%$ weight fraction at $323 \mathrm{~K})$. Another unidentified impurity phase appears above $\sim 1000 \mathrm{~K}$ that may be a product of the reaction of $\mathrm{AlF}_{3}$ and the quartz capillary. However, the $\alpha-\mathrm{AlF}_{3}$ peaks are much more intense than these impurity peaks. 

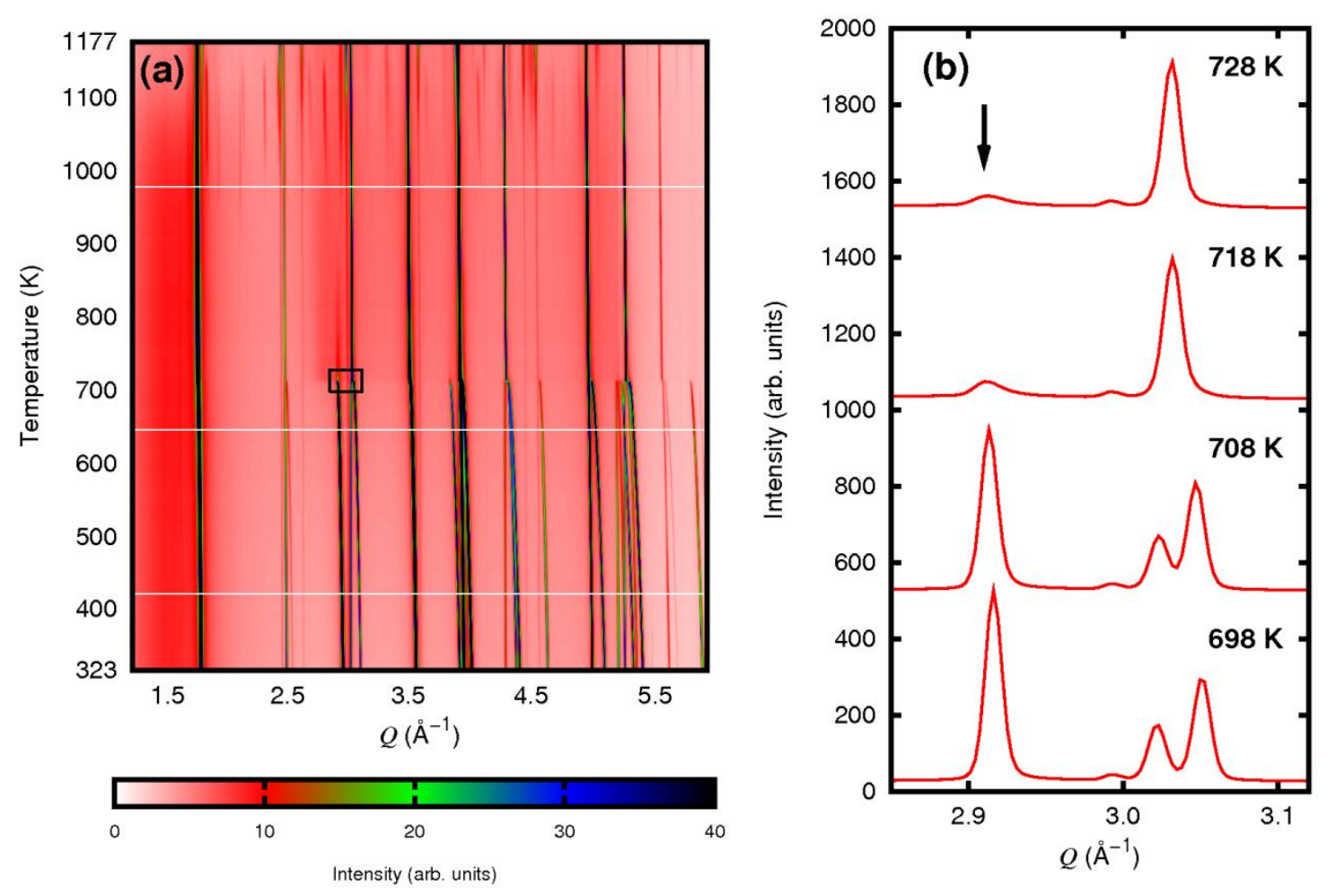

Figure 2. (a) Variable temperature (323 to $1177 \mathrm{~K}$ ) powder $\mathrm{X}$-ray diffraction patterns for $\alpha-\mathrm{AlF}_{3}$, displayed in order of collection from bottom to top. The horizontal white lines are from frames that were lost during data collection. (b) X-ray diffraction data for $\alpha-\mathrm{AlF}_{3}$ at selected temperatures, showing the presence of a diffuse peak (marked by an arrow) that is incommensurate with the $\mathrm{AlF}_{3}$ unit cell. These data are taken from the portion of (a) enclosed by a small box.

The temperature dependence of both unit cell volume per formula unit and volume CTE of $\alpha$ $\mathrm{AlF}_{3}$ are plotted in Figure 3. When heated from ambient temperature, rhombohedral $\alpha-\mathrm{AlF}_{3}$ exhibits strongly positive volume thermal expansion $\left(\alpha_{V}(500 \mathrm{~K}) \sim 86 \mathrm{ppm} \cdot \mathrm{K}^{-1}\right)$ as it unfolds. Above the first-order phase transition, cubic $\alpha-\mathrm{AlF}_{3}$ continues to expand but with a much lower CTE $\left(\alpha_{V}(900 \mathrm{~K}) \sim 25 \mathrm{ppm} \cdot \mathrm{K}^{-1}\right)$. The thermal expansion of rhombohedral $\alpha-\mathrm{AlF}_{3}$ is strongly anisotropic (Figure 4). The $a$-axis displays strong positive thermal expansion, while the CTE 
along the $c$-axis is close to zero. This anisotropy occurs because the rhombohedral-to-cubic phase transition involves rotation of the $\mathrm{AlF}_{6}$ octahedra around their three-fold axes, which are parallel to the crystallographic $c$-axis. Based on the temperature dependence of $c / a$ (Figure 5a), we estimate the phase transition temperature as $713 \mathrm{~K}$, which is consistent with the temperature reported in another XRD study $(715 \pm 10 \mathrm{~K})^{21}$ but slightly lower than that measured by differential scanning calorimetry $(725 \mathrm{~K})^{17}$ and XRD $(741 \pm 10 \mathrm{~K})$ in other studies. ${ }^{20}$

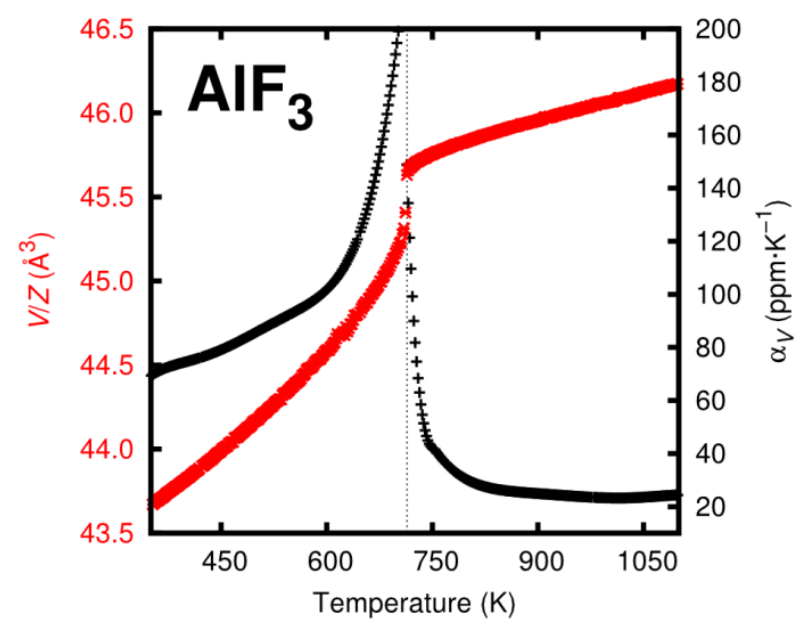

Figure 3. Temperature dependence of the unit cell volume per formula unit $(V / Z$, red $x)$ and volume CTE $(\alpha$, , black +$)$ of $\alpha-\mathrm{AlF}_{3}$. Unit cell volumes were extracted from Rietveld analyses of PXRD data, and $\alpha_{V}$ were estimated from polynomial fits to the $V / Z$ vs. $T$ data. The dashed line indicates the estimated phase transition temperature (713 K). 

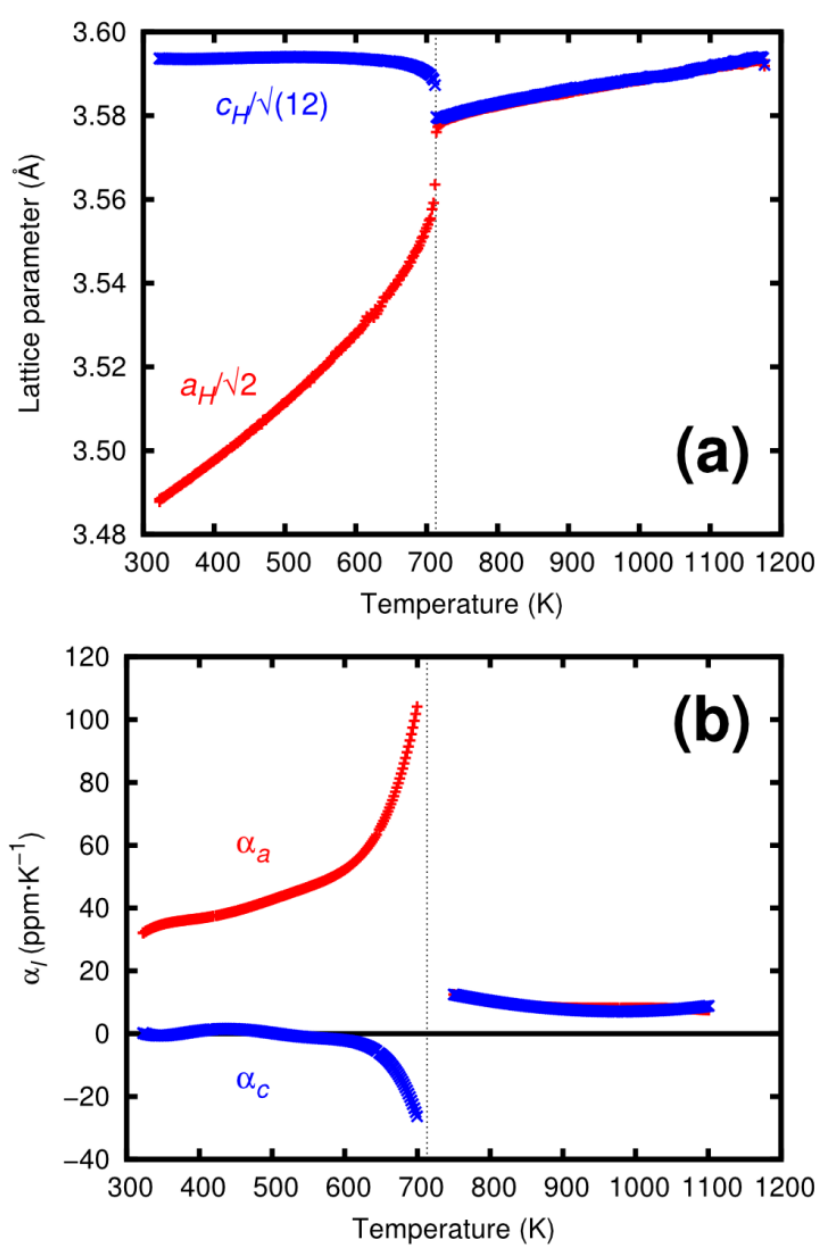

Figure 4. (a) Temperature dependence of the lattice parameters of $\alpha-\mathrm{AlF}_{3}$. Hexagonal values $a_{H}$ and $c_{H}$ are reduced to their cubic equivalents by the indicated factors. A rhombohedral model was used for all temperatures, even above the phase transition, where $a_{H}$ and $c_{H}$ refine to the same value. (b) Temperature dependence of the linear CTEs; $\alpha_{a}$ and $\alpha_{c}$ are significantly different below the phase transition temperature but equal above it. 

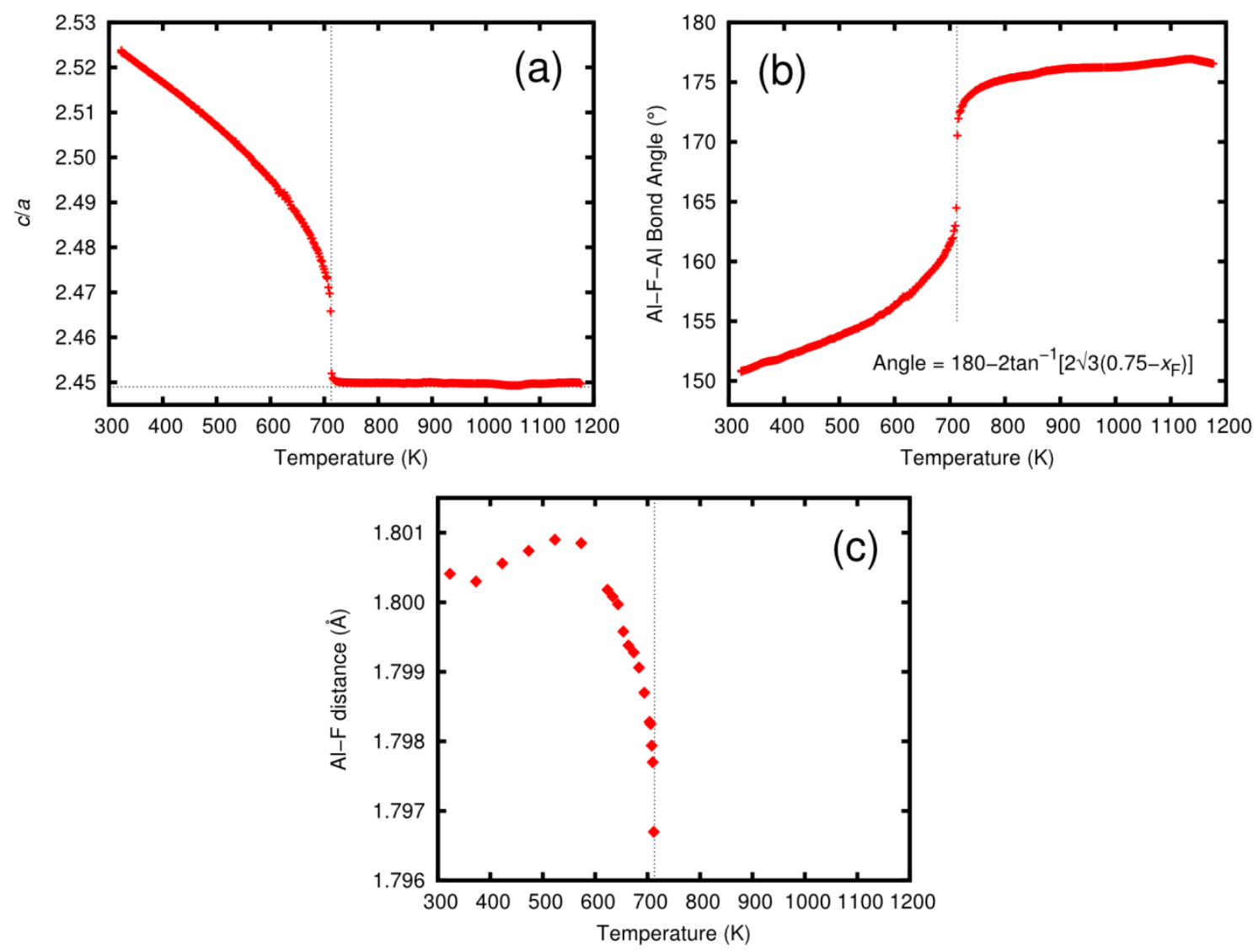

Figure 5. Temperature dependence of (a) the ratio of lattice constants $c$ and $a$ (hexagonal cell), which was used to estimate the phase transition temperature of $713 \mathrm{~K}$ (marked in each panel by a vertical dashed line), (b) the estimated $\mathrm{Al}-\mathrm{F}-\mathrm{Al}$ bond angle, related to the $x$-coordinate of $\mathrm{F}$ $\left(x_{\mathrm{F}}\right)$ by the equation shown, and (c) the $\mathrm{Al}-\mathrm{F}$ distance. The expected value for $c / a$ in the cubic phase is 2.449, as indicated by a horizontal dashed line in (a), and the expected value of $x_{\mathrm{F}}$, in the absence of any disorder, in the cubic phase is 0.75 , which would lead to an $\mathrm{Al}-\mathrm{F}-\mathrm{Al}$ bond angle of $180^{\circ}$.

As shown in Figure 2, there are some diffuse peaks at approximately the positions of the rhombohedral supercell reflections that persist to temperatures well above the phase transition, and the transition is accompanied by a significant increase in "background" scattering; it is notable that these diffuse peaks are incommensurate, in that they do not index on the unit cell. The most prominent of the diffuse peaks appears at $Q \sim 3.0 \AA^{-1}$ (marked by an arrow in Figure 
2b), which, prior to the phase transition, corresponds approximately to the rhombohedral $\left(\begin{array}{lll}2 & 1 & 0\end{array}\right)$ Bragg peak; its area drops significantly with increased temperature, and its width is greater than that of the Bragg peaks and increases sharply immediately above the phase transition temperature, $713 \mathrm{~K}$ (Supporting Information, Figure S2). In addition, the peak shifts to higher angles on heating, counter to the PTE of $\mathrm{AlF}_{3}$. These diffuse peaks and the jump in background due to diffuse scattering suggest the presence of short-range structural distortions in the cubic phase. Such distortions have been noted previously. Chupas et al. also observed weak superlattice PXRD peaks persisting past the phase transition and calculated an $\mathrm{Al}-\mathrm{F}-\mathrm{Al}$ bond angle of $175.8^{\circ}$ at $723 \mathrm{~K}$, suggesting a small degree of local rhombohedral distortion. ${ }^{16}$ Both molecular dynamics simulations ${ }^{19}$ and pair distribution function analysis ${ }^{20}$ confirmed the presence of bent $\mathrm{Al}-\mathrm{F}-\mathrm{Al}$ bonds and tilted octahedra in the cubic structure.

Our attempts to fit a cubic model with a split F site to the data above the phase transition, in the manner of Chupas et $a l,{ }^{20}$ yielded unrealistic values for the $\mathrm{F}$ position and atomic displacement parameters (ADP), perhaps because the $Q$ range of our data was much smaller than that used by the previous authors $\left(Q_{\max } \sim 6\right.$ and $\sim 13 \AA^{-1}$, respectively). However, by using the primitive rhombohedral model at all temperatures, where the variable $x_{\mathrm{F}}$ is partly accounting for disorder in the cubic phase, we estimated $\mathrm{Al}-\mathrm{F}-\mathrm{Al}$ bond angles that reasonably matched the previous observations ${ }^{16,19,20}$ (Figure 5b). Such an approach must be treated with caution, due to the incomplete nature of the model and possible correlations involving ADPs. The apparent AlF-Al bond angle estimated from the rhombohedral model increases steadily with temperature to at least $1100 \mathrm{~K}$, which is consistent with our observation of quite strong PTE in cubic $\alpha-\mathrm{AlF}_{3}$. Furthermore, the $\mathrm{Al}-\mathrm{F}$ distance does not vary greatly with temperature (300 to $\sim 600 \mathrm{~K}$ ) (Figure 5c), indicating the presence of rigid $\mathrm{AlF}_{6}$ octahedra, with the positive thermal expansion 
principally driven by coupled rotation of these octahedra. However, beginning at $\sim 600 \mathrm{~K}$, as the phase transition is approached, the $\mathrm{Al}-\mathrm{F}$ distance appears to decrease with temperature. This behavior presumably reflects the presence of static/dynamic disorder rather than an actual decrease in the instantaneous $\mathrm{Al}-\mathrm{F}$ distance. Zhao et al. reported similar, although more pronounced, behavior for the $\mathrm{Mg}-\mathrm{F}$ distance in the perovskite $\mathrm{NaMgF}_{3} .{ }^{26}$

The strong PTE of cubic $\alpha-\mathrm{AlF}_{3}$ stands in contrast to the behavior of cubic $\mathrm{ScF}_{3}$ (strong NTE) and $\mathrm{TiF}_{3}$ (low PTE or low NTE depending upon temperature). However, there are other examples of PTE in the cubic $\mathrm{ReO}_{3}$ family; for example, cubic $\mathrm{NbO}_{2} \mathrm{~F}$ expands on heating between 20 and $500 \mathrm{~K} \cdot{ }^{79}$ Based on our local structural study of $\mathrm{TaO}_{2} \mathrm{~F}^{8}$, we proposed that the PTE in $\mathrm{NbO}_{2} \mathrm{~F}$ may arise from the presence of bent $\mathrm{Nb}-(\mathrm{O} / \mathrm{F})-\mathrm{Nb}$ links whose bond angles increase on heating. A similar phenomenon may be involved in the quite strong PTE of cubic $\mathrm{AlF}_{3}$.

\section{CONCLUSION}

The volume thermal expansion of $\alpha-\mathrm{AlF}_{3}$ is strongly positive above ambient temperature, where the structure is rhombohedral. After transforming to the cubic $\mathrm{ReO}_{3}$-type structure at 713 $\mathrm{K}$, the volume CTE drops by fourfold but remains positive to at least $1177 \mathrm{~K}$. The positive thermal expansion of the cubic phase may be partially due to the presence of local octahedral tilts and an associated increase in the average $\mathrm{Al}-\mathrm{F}-\mathrm{Al}$ bond angle on heating.

\section{SUPPORTING INFORMATION}

The following are available as supporting information: sample Rietveld fit to data collected at $323 \mathrm{~K}$; temperature dependence of structural parameters for $\alpha-\mathrm{AlF}_{3}$; temperature dependence of 
the diffuse peak at $Q \sim 3.0 \AA^{-1}$; Rietveld refinement results at selected temperatures; primitive rhombohedral lattice constants and $x$-coordinate of $\mathrm{F}$ for $\alpha-\mathrm{AlF}_{3}$ between 323 and $1177 \mathrm{~K}$.

\section{ACKNOWLEDGEMENTS}

A.P.W. is grateful for support from the National Science Foundation under grant DMR-0905842.

Use of the Advanced Photon Source at Argonne National Laboratory was supported by the United States Department of Energy, Office of Science, Office of Basic Energy Sciences, under Contract No. DE-AC02-06CH11357. The authors are also grateful for the experimental support of G. J. Halder at beamline 17-BM-B of the Advanced Photon Source.

\section{REFERENCES}

(1)Miller, W.; Smith, C. W.; Mackenzie, D. S.; Evans, K. E. J Mater Sci 2009, 44, 5441.

(2)Tao, J. Z.; Sleight, A. W. J. Solid State Chem. 2003, 173, 442.

(3)Chatterji, T.; Henry, P. F.; Mittal, R.; Chaplot, S. L. Phys. Rev. B 2008, 78, 134105.

(4)Chatterji, T.; Hansen, T. C.; Brunelli, M.; Henry, P. F. Appl. Phys. Lett. 2009, 94, 241902.

(5)Rodriguez, E. E.; Llobet, A.; Proffen, T.; Melot, B. C.; Seshadri, R.; Littlewood, P. B.; Cheetham, A. K. J. Appl. Phys. 2009, 105, 114901.

(6)Dapiaggi, M.; Fitch, A. N. J. Appl. Cryst. 2009, 42, 253.

(7)Tao, J. Z.; Sleight, A. W. J. Solid State Chem. 2003, 173, 45.

(8)Morelock, C. R.; Greve, B. K.; Cetinkol, M.; Chapman, K. W.; Chupas, P. J.; Wilkinson, A. P. Chem. Mater. 2013, 25, 1900.

(9)Wilkinson, A. P.; Josefsberg, R. E.; Gallington, L. C.; Morelock, C. R.; Monaco, C. M. J. Solid State Chem. 2014, 213, 38.

(10) Greve, B. K.; Martin, K. L.; Lee, P. L.; Chupas, P. J.; Chapman, K. W.; Wilkinson, A. P. J. Am. Chem. Soc. 2010, 132, 15496.

(11) Morelock, C. R.; Greve, B. K.; Gallington, L. C.; Chapman, K. W.; Wilkinson, A. P. J. Appl. Phys. 2013, 114, 213501.

(12) Morelock, C. R.; Gallington, L. C.; Wilkinson, A. P. Chem. Mater. 2014, 26, 1936.

(13) Daniel, P.; Bulou, A.; Rousseau, M.; Nouet, J.; Leblanc, M. Phys. Rev. B 1990, 42, 10545.

(14) Kennedy, B. J.; Vogt, T. Mater. Res. Bull. 2002, 37, 77.

(15) Mogus-Milankovic, A.; Ravez, J.; Chaminade, J. P.; Hagenmuller, P. Mater. Res. Bull. 1985, 20, 9 .

(16) Chupas, P. J.; Ciraolo, M. F.; Hanson, J. C.; Grey, C. P. J. Am. Chem. Soc. 2001, 123, 1694.

(17) Daniel, P.; Bulou, A.; Rousseau, M.; Nouet, J.; Fourquet, J. L.; Leblanc, M.; Burriel, R. J. Phys.: Condens. Matter 1990, 2, 5663.

(18) Chen, Y.-R.; Perebeinos, V.; Allen, P. B. Phys. Rev. B 2004, 69, 054109. 
(19) Chaudhuri, S.; Chupas, P. J.; Wilson, M.; Madden, P.; Grey, C. P. J. Phys. Chem. B 2004, 108, 3437.

(20) Chupas, P. J.; Chaudhuri, S.; Hanson, J. C.; Qiu, X.; Lee, P. L.; Shastri, S. D.; Billinge, S. J. L.; Grey, C. P. J. Am. Chem. Soc. 2004, 126, 4756.

(21) Ravez, J.; Mogus-Milankovic, A.; Chaminade, J. P.; Hagenmuller, P. Mater. Res. Bull. 1984, 19, 1311.

(22) Chupas, P. J.; Chapman, K. W.; Kurtz, C.; Hanson, J. C.; Lee, P. L.; Grey, C. P. J. Appl. Cryst. 2008, 41, 822 .

(23) Hammersley, A. P.; Svensson, S. O.; Hanfland, M.; Fitch, A. N.; Hausermann, D. High Pressure Res. 1996, 14, 235.

(24) Larson, A. C.; Von Dreele, R. B. General Structure Analysis System (GSAS), Los Alamos National Laboratory, 2000.

(25) Toby, B. J. Appl. Cryst. 2001, 34, 210.

(26) Zhao, Y.; Weidner, D. J.; Parise, J. B.; Cox, D. E. Phys. Earth Planet In. 1993, 76, 1. 